Marta Wylot"

\title{
DIE TEXTSORTE GLOSSE IN DIACHRONISCHER AUFFASSUNG
}

\section{Einleitung}

Die Presse ist die vierte Macht. Sie kann die Entscheidungen der Politiker, der Gesellschaft, der Einrichtungen und Institutionen beeinflussen. Sie erfreut sich großer Beliebtheit, denn jeden Tag werden Millionen verschiedener Zeitungen und Zeitschriften gedruckt, gekauft oder online gelesen. Manche Zeitungen und Zeitschriften sind meinungsbildend und sie können auf die öffentliche Meinung Einfluss haben. Um diesen Zweck zu erreichen, können sich die Journalisten verschiedener Textsorten bedienen, wobei jede Textsorte ein anderes Ziel und einen anderen Charakter hat und dadurch die Anwendung anderer stilistischen Mittel erzwingt. Eine der Textsorten, der sich die Journalisten bedienen können ist die Glosse. Das Ziel des Artikels liegt darin, den Begriff Glosse zu definieren und zu beschreiben, wie sich diese Textsorte diachron geändert hat.

\section{Zur Definition der Glosse}

Am Anfang dieses Artikels soll die Textsorte definiert werden. Im 19. Jahrhundert wurde mit dem Begriff Glosse sowohl die Textsorte als auch ein Ressort der Zeitung oder Zeitschrift bezeichnet (Pietrzak 2013, S. 10). In der polnischen Literatur wird hauptsächlich die Bezeichnung felieton verwendet, mit der sowohl die Textsorte als auch ein Teil einer Zeitung beziehungsweise Zeitschrift gemeint wird. Die deutsche Bezeichnung Feuilleton bezieht sich auf ein Ressort der Zeitung oder Zeitschrift. Reus (1999, S. 7) unterstreicht, dass heutzutage der Begriff Feuilleton nur als eine Bezeichnung des Ressorts für das Kulturelle gebraucht wird. Das, was in der polnischen Literatur unter dem Begriff felieton gefasst wird, wird in der deutschen Literatur als Glosse bezeichnet.

* Marta Wylot M. A., Universität Łódź, Philologische Fakultät, Lehrstuhl für Deutsche und Angewandte Sprachwissenschaft, 90-236 Łódź, Pomorska 171/173. 
Innerhalb des Resorts kommen viele Textsorten vor. Für die Textsorte Glosse gibt es viele Definitionen. Die Glosse wird immer als eine kurze Form betrachtet, jedoch weisen die Definitionen auf viele andere Aspekte hin. Es wurde eine Fragebogenaktion bei 121 bundesrepublikanischen Tageszeitungen mit Vollredaktion durchgeführt, die zum Ziel hatte, genau zu bestimmen, was Glosse ist und wie sie aufgebaut werden soll. Die Mehrheit der Redaktionen definiert die Glosse als „sarkastischen, satirischen Kurzkommentar, als spöttisch-polemische Randbemerkung in feuilletonistischer Form" (Nowag/Schalkowski 1998, S. 183). 24\% der Redaktionen versteht unter dem Begriff Glosse ,einen ersten Kurzkommentar ohne satirische, sarkastische, polemische Komponenten“". 69\% der Redakteure charakterisieren die Glosse als humoristisch, unterhaltsam und herzerquickend und nach 31\% ist die Glosse aggressiv, kämpferisch und verletzend.

Es wurden auch Untersuchungen zum Feuilleton als Ressorts durchgeführt, und zwar wurde versucht, die Themenbereiche zu bestimmen, denen die in diesem Teil enthaltenen Texte gewidmet werden. $\mathrm{Zu}$ diesen Themenbereichen gehören vor allem in Regionalzeitungen: Theater, Belletristik und bildende Kunst. Das sind ungefähr $74 \%$ aller Texte. Weitere $11 \%$ beschäftigen sich mit Film und anderen Medien (Reus 1999, S. 24f.). Also es wird ein großer Teil der Texte der Kunst gewidmet. In den Wochenendebeilagen sieht die Tendenz anders aus. 27\% der Texte befassen sich mit Film und Medien und 58\% mit weiteren Kulturthemen, Medizin, Geschichte und Gesellschaft. Dabei bemerkt Reus ein wichtiges Phänomen. Je mehr die in den Glossen behandelten Themen sich dem Alltag nähern, desto seltener werden die Texte mit dem Strich von dem übrigen Text getrennt. Sehr selten ist es der Fall, dass sich diese Texte mit den Themenbereichen wie Sport oder Wirtschaft befassen (vgl. Reus 1999, S. 34).

Fricke und Weimar (1997, S. 727f.) weisen auf die Dreiteilung der Glosse als „Instrument der Texterschließung bezogen auf das Einzelwort oder einen umfänglicheren Textzusammenhang", als ein Gedicht, in dem ein vorgegebenes Thema zitiert oder paraphrasiert wird (S. 728) oder als „kurzen journalistischen Meinungstext" (S. 30) hin.

Lüger (1995, S. 137) betont, dass die Glosse zum Ziel hat, aus den widerstreitenden Meinungen eine Stellung konsensfähig zu machen. Außerdem muss sich der Glossist auf ein bestimmtes Problem konzentrieren; er kann die Glosse nicht mit zusätzlichen Informationen überfluten.

Nach Linden/Bleher (2002) ist die Glosse eine Art des Kommentierens mit spürbarer Ironie, die versteckt ist (S. 19, 98f.). Sie stellen fest, dass die Wurzeln der Textsorte Glosse in der Satire zu finden sind. Aber grundsätzlich liegt der Glosse das Textmuster Kommentar zugrunde: Zuerst muss etwas passieren, was beschrieben und kommentiert wird, dann wird die Glosse verfasst, die sich auf das Geschehene bezieht und sich solcher Mittel wie Hyperbel bedient. Zwar sind Ironie und Satire literarische Phänomene, aber die Glosse hat sich in der Journalistik platziert. Sie ist dann zu verfassen, wenn das aktuelle Ereignis von einem 
emotionalen Reiz begleitet wird. Linden/Bleher (2002, S. 87) behaupten, dass die Glosse in folgenden Fällen geschrieben wird, wenn:

- die Nachricht oder das Geschehen etwas Komisches in sich hat,

- der Nachricht oder dem Geschehen zu große Bedeutung zugeschrieben wird,

- die Personen zu bedeutsam eingeschätzt sind,

- die dargestellte These allgemein gültig und gut sein soll, ist aber nicht so. Nowag/Schalkowski (1998, S. 184) nennen andere Motive und Ziele, die dem Verfassen der Glosse zu Grunde liegen. Dazu gehören:

- Kritik und Meinungsäußerung,

- Änderung der Meinung,

- Angriff, Verspottung, Ausdruck der Schadenfreude,

- Anregung zum Nachdenken,

- Belustigung.

Je nach Form unterscheiden sie folgende Arten von Glosse:

- die karikierende Glosse,

- die ironische, zynische und sarkastische Glosse,

- die Vexierglosse,

- die Zitatglosse,

- die Sprachglosse,

- die groteske Glosse,

- die literarisch-fiktive-satirische Glosse.

Die Glosse ist, wie oben angedeutet, der Struktur nach dem Kommentar ähnlich; sie ist argumentativ, polemisch, vergleichend, analytisch und konstruktiv. Nowag/ Schalkowski stellen folgendes fest (1998, S. 184): „Der Kommentar umfasst die Glosse, die Glosse jedoch nicht den Kommentar. Die Glosse verhielt sich zum Kommentar wie der Igel zum Tier. [...] Kommentar ist der Oberbegriff". Die Autoren definieren die Glosse als ,einen Kurzkommentar spöttisch-ironischen, satirischen, sarkastisch-bitteren, grotesken Inhalts. Die Glosse ist also eine Spezialform des Kommentars, eben ein Kommentar, der sich der Mittel des Spotts, der Ironie, der Satire, des Sarkasmus, der Groteske bedient“. Neben der Glosse gehören auch der Kommentar und der Leitartikel zu den meinungsorientierten Texten, die einen Sachverhalt beschreiben. Der Kommentar und der Leitartikel nehmen dazu Stellung auf eine diplomatische Art und Weise, die Glosse dagegen spielt mit Assoziationen, fingiert Situationen, kombiniert Zitate, überspringt die räumlichen und zeitlichen Grenzen, missachtet die Gesetze von Logik, Kausalität und Alltagserfahrung. Beide Texte sind auch kritisch und wertend. Der Unterschied zwischen diesen Textsorten liegt darin, wie sie den Sachverhalt kommentieren. Der Kommentar stellt den Sachverhalt unter dem Gesichtspunkt der Wahrheit, der gesellschaftlich gültigen Normativität, des Rechts, des Grundgesetzes sowie der universellen Ideale dar. Die Glosse widerspiegelt den Sachverhalt als eine Form der Utopie, in der die menschlichen Tugenden eine große Rolle spielen (ebd., S. 223, 230). 
Da Glosse dem Kommentar ähnlich ist, sieht der Schaffensprozess des Kommentars und der Glosse in der Anfangsphase analog aus. Linden/Bleher (2002, S. 116) nennen zehn Schritte der Entstehung einer Glosse:

- das Passieren des Geschehens oder des Erlebnisses,

- das Auslösen eines emotionalen Reizes, einer emotionalen Reaktion (z.B. Wut oder Lachen),

- die Formulierung einer These, grundsätzlich einer kritischen These,

- die Ideensammlung, Recherche,

- der Verzicht auf die Form des Kommentars,

- die Sammlung der Assoziationen,

- die Übertreibung,

- die Wahl des Rollenspiels,

- der Aufbau der Textstruktur, der Pointe, der Schlussfolgerungen,

- die sprachliche Ausgestaltung.

Im Falle der Glosse fällt es schwieriger, den Text auszuwerten, „weil offensichtlich keine allgemein gültige Ansicht darüber besteht, was unter Glosse firmiert werden kann" (Linden/ Bleher 2002, S. 8). Doch es wird ziemlich genau formuliert, was die Glosse ist. Die Autoren führen folgende Definition der Glosse an: „spöttische Randbemerkung zu einem aktuellen Thema“, wobei sie bemerken, dass für die Glosse auch andere Eigenschaften kennzeichnend sind wie klare Struktur und Techniken, die den komischen Effekt hervorrufen sollen. Dabei stellt sich die Frage, was die Glosse darf. Eckhard Henscheid (1996, S. 18) weist darauf hin, dass die Glosse alles darf. Dies kann die Gefahr mit sich bringen, dass die Glosse die Ehre der in den Glossen angesprochenen Personen verletzen kann. Sie können sich auf die Paragraphen 185 bis 187 des Strafgesetzbuches berufen. Diesen Vorschriften wird aber der 5. Artikel des Grundgesetzes entgegengesetzt, der für die Freiheit der Kunst und der Meinungsäußerung plädiert. In dieser widersprüchlichen Regelung wird die Frage nach den moralischen Grenzen gestellt. Die Glosse moralisiert, was an die Wurzeln in der aufklärerischen Tradition des Journalismus anknüpft. Die Textsorte setzt die Privilegierten den Unterdrückten, die Mächtigen den Schwachen entgegen.

\section{Zur Geschichte der Glosse}

In der Fachliteratur wird allgemein angegeben, dass die Wurzeln dieser Textsorte in Frankreich im 19. Jahrhundert liegen, was aber nach Stöber (2000, S. 183) nur teilweise korrekt ist. Die aus dem Französischen stammende Bezeichnung Feuilleton heißt Blättchen. Sie weist darauf hin, dass die ersten Glossen auf dem unteren Seitendrittel oder -viertel unter dem Strich platziert waren. Mit dem Begriff Feuilleton wurde darüber hinaus das Buchbesprechungsteil einer Zeitung bereits im 18. Jahrhundert genannt. So hat Julien Louis de Geoffroy auch seine Theaterrubrik im „Journal des Débats“ betitelt. Die ersten feuilletonistischen Texte sind also im 18. 
Jahrhundert in Zeitungen und Zeitschriften publiziert worden (Stöber 2000, S. 183; Pietrzak 2013, S. 31f.). In der englischen Journalistik waren es die Texte von Joseph Addison und Richard Stecle in der Zeitschrift "Spectator", deswegen scheint die Geschichte mit „Journal des Débats“ eine Legende zu sein. Dieser Zeitung kann diese Textsorte nur den Namen verdanken. Als Vorläufer der Feuilletonisten gelten die Journalisten der Französischen Revolution sowie Heinrich Heine oder Aleksander Puschkin. In Polen sind als Vorläufer dieser Textsorte Publizisten angesehen, die ihre Texte in „Monitor" und „Zabawy Przyjemne i Pożyteczne“"veröffentlicht haben.

Stöber (2000, S. 184ff.) betont, dass es ursprünglich schwierig war, diese Textsorte zu definieren. Dieses Kriterium, dass diese Textsorte unter dem Strich platziert werden soll, ist nicht ausreichend, weil auch die Leserbriefe dort zu finden waren. Im 20. Jahrhundert wurden in dem Ressort Feuilleton Romane gedruckt, um die Gewinne aus dem Verkauf zu erhöhen. In der kommunistischen Presse hatte die Veröffentlichung der Romane in diesem Ressort ein anderes Ziel und zwar Erziehung der Bürger. Als erste Form der Glosse wurde die Buchbesprechung betrachtet. Die Zeitungen und Zeitschriften, die die Kritiken veröffentlicht haben, haben im 17. und 18. Jahrhundert eine große Bedeutung gespielt. Zuerst wurden die Buchkritiken verfasst. Die ersten Musikkritiken, die die journalistischen Standards erfüllt haben, wurden in den musikalischen Fachzeitschriften im 18. Jahrhundert veröffentlicht. 1907 wurde die erste eigenständige Filmzeitschrift publiziert, in der die Kritiken der Filme geschrieben wurden (Stöber 2000, S. 1836ff.). Den Journalisten, die die ersten feuilletonistischen Texte verfasst haben, wurde vorgeworfen, dass sie käuflich seien. Im 19. Jahrhundert wurden die ersten Glossen verfasst, die den politischen Themen gewidmet waren, da die politischen Kommentare in dieser Zeit entweder verboten oder stark reglementiert waren. Auch im 19. Jahrhundert wurden die ersten stark politisch geprägten Gedichte verfasst. Das Regime hat dieses Phänomen bald bemerkt, deswegen haben sich die Verfasser der Mehrdeutigkeit bedient, um die Konsequenzen zu vermeiden. Jakoby (1998, S. 4) meint, dass die Kritik des Alltags in den Zeiten der Zensur in Form von Romanen und Gedichten versteckt war. Das Spektrum der Themen, die die Glossen betroffen haben, wurde auch immer breiter. In derselben Zeit haben die Glossen auch eine andere Rolle gespielt (Stöber 2000, S. 1836ff.). Die Wissenschaftler, wie z.B. Justus Liebig, haben in diesen Texten ihre wissenschaftlichen Ideen vermittelt (Stöber 2000, S. 189). Diese Funktion spielt die Glosse gegenwärtig nicht mehr. Auch im 19. Jahrhundert haben die Feuilletonisten angefangen, ihre Texte mit Pseudonymen zu unterschreiben (Pietrzak 2013, S. 33).

Die ersten Versuche der Begriffsbestimmung der Glosse in Polen haben auch im 19. Jahrhundert stattgefunden (Pietrzak 2013, S. 34f.). Es waren vor allem Publizisten, die sich kritisch über die Glosse und über Feuilletonisten geäußert haben. Die erste Definition dieser Textsorte wurde 1851 von Cyprian Kamil Norwid formuliert, der sie vor allem als ,politischen Lyrismus“ verstand. Eine genauere Definition wurde in „Encyklopedia powszechna“ von Orgelbrand formuliert. Mit der Glosse wurde ein Ressort in einer Zeitung oder Zeitschrift bezeichnet, der 
unter dem Strich platziert war, in dem am häufigsten Romane, Kritiken, literarische und wissenschaftliche Artikel veröffentlich wurden. Sie hatte auch eine gesellschaftliche Rolle gespielt, indem sie einen Einblick in das städtische Leben in einer witzigen Form verschafft hat. Nach Pietrzak (2013, S. 50) dient die Glosse der Unterhaltung, aber in erster Linie sollte sie die Leser erziehen, das Gute loben und das Böse tadeln. In den 70er Jahren des 19. Jahrhunderts wurden sich immer mehr Autoren der Textsorte Glosse bewusst. In dieser Zeit haben Wacław Szymanowski, Adam Wiślicki, Henryk Sienkiewicz und Bolesław Prus angefangen, Glossen zu schreiben und zu veröffentlichen (Pietrzak 2013, S. 37ff.).

Oleze (1998, S. 33) bemerkt, dass im Ressort Feuilleton der „Kölnischen Zeitung" Plaudereien, Skizzen, Erzählungen und Romanabdrücke veröffentlicht wurden.

Heute werden die Glossen für große Tageszeitungen von Redakteuren, freien Journalisten, festen Freien, Gastbeiträgern oder Philologen geschrieben (Wildehann 2006, S. 30).

\section{Die Glosse vor Jahren}

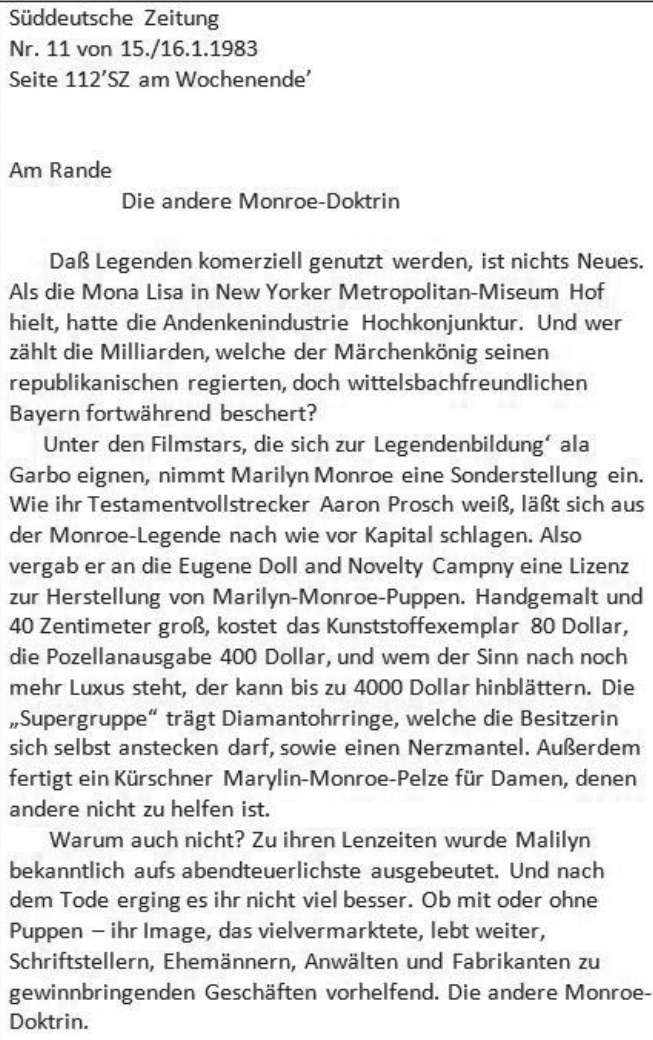

Süddeutsche Zeitung Nr. 11 vom 15./16.01.1983, S. 112 


\section{Die Glosse von heute}

\section{a DIECWELT ,

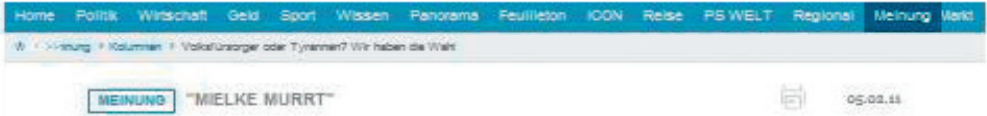 \\ Volksfürsorger oder Tyrannen? Wir haben die Wahl}

So schnell kann es gehen: eben noch anerkannter Staatschef, nun verschmähter Tyrann. André Mielke fragt sich, ob das auch in Berlin passieren könnte.

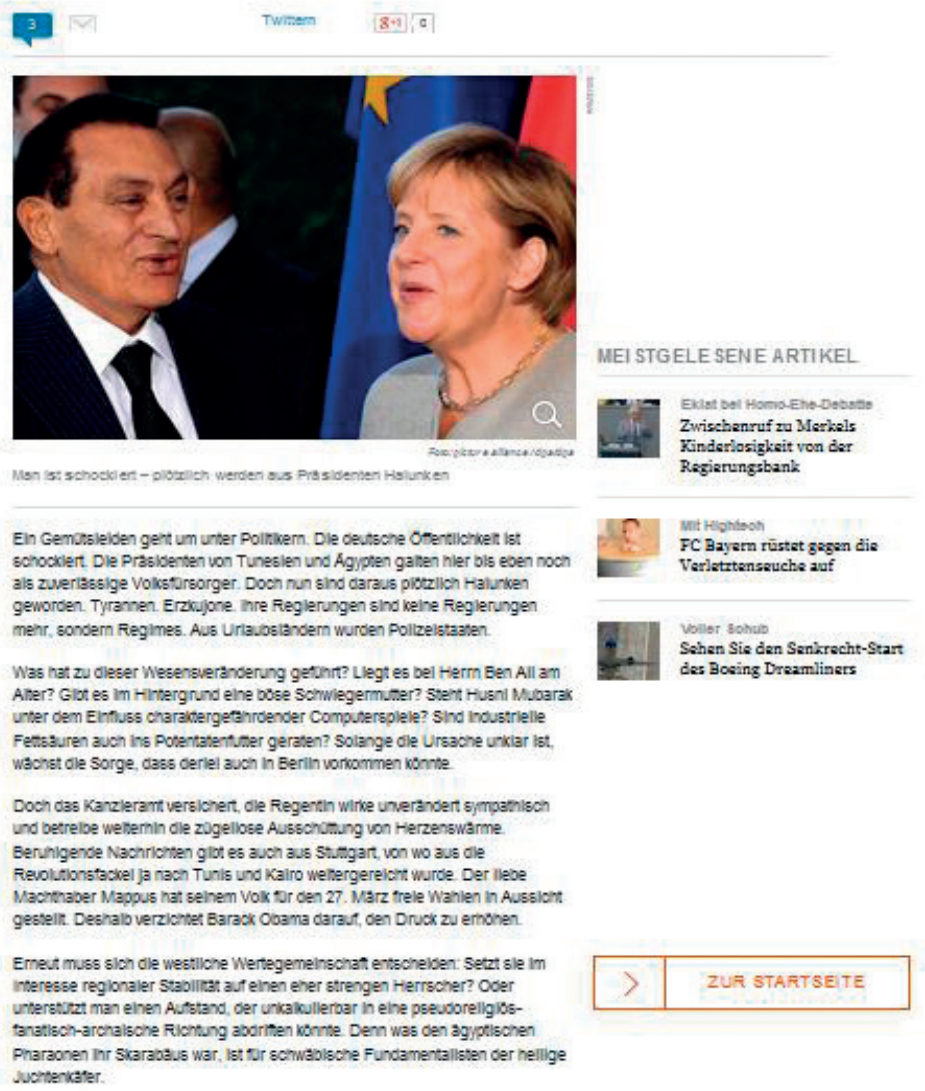

http://www.welt.de/debatte/kolumnen/Mielke-murrt/article12455820/Volksfuersorger-oderTyrannen-Wir-haben-die-Wahl.html

Um das aktuelle Bild der Textsorte zu skizzieren, werden ihre Eigenschaften anhand des Textes „Volksfürsorger oder Tyrannen? Wir haben die Wahl” aus der Tageszeitung ,Die Welt‘ dargestellt. Die Glosse wurde am 5. Februar 2011 veröffentlicht. 
Der Text bezieht sich auf die letzten Ereignisse in Tunesien und Ägypten. Die Präsidenten beider Länder haben eine neue Ordnung eingeführt, die der Gesellschaft nicht gefällt.

Der Text befindet sich in der festen Rubrik der Tageszeitung - „Mielke murrt“. Die Glosse ist kurz. Der Text beginnt mit der Überschrift „Volksfürsorger oder Tyrannen? Wir haben die Wahl". Danach wird der Leser in das Thema eingeführt und am Ende kommt die Pointe: „Denn was den ägyptischen Pharaonen ihr Skarabäus war, ist für schwäbische Fundamentalisten der heilige Juchtenkäfer". Mit der Pointe wird das Kriterium der Intertextualität erfüllt, weil die Pointe eine Art der Anspielung ist, die das Vorwissen des Lesers erfordert.

Der Text enthält viele Eigenschaften, die für die Glosse typisch sind. Es kommen viele Ausdrücke vor, die sich vom Schreibstil unterscheiden: „Doch nun sind daraus plötzlich Halunken geworden. Tyrannen. Erzkujone“, was den ironischen Effekt hervorruft. Außerdem tauchen im Text viele rhetorische Fragen auf: „Was hat zu dieser Wesensveränderung geführt? Liegt es bei Herrn Ben Ali am Alter? Gibt es im Hintergrund eine böse Schwiegermutter? Steht Husni Mubarak unter dem Einfluss charaktergefährdender Computerspiele?"“ Im Text wurden die Wörter auch metaphorisch verwendet: „Beruhigende Nachrichten gibt es auch aus Stuttgart, von wo aus die Revolutionsfackel ja nach Tunis und Kairo weitergereicht wurde“. Die Anzeichen der Ironie sind in folgenden Sätzen zu sehen: „Erneut muss sich die westliche Wertegemeinschaft entscheiden: Setzt sie im Interesse regionaler Stabilität auf einen eher strengen Herrscher? Oder unterstützt man einen Aufstand, der unkalkulierbar in eine pseudoreligiös-fanatisch-archaische Richtung abdriften könnte“".

Der Autor kritisiert die Regierenden in Tunesien und Ägypten; sie werden verspottet. Er äußert dabei seine eigene Meinung, dass sich die Geschichte auch in Berlin wiederholen kann. Der Text regt zum Nachdenken an. Jeder Leser sucht nach Ähnlichkeiten zwischen Tunesien, Ägypten und Deutschland. Dabei ist die Übertreibung des Autors zu sehen: „Die deutsche Öffentlichkeit ist schockiert oder Solange die Ursache unklar ist, wächst die Sorge, dass derlei auch in Berlin vorkommen könnte“.

Die Glosse erfüllt vor allem die Appellfunktion. Da die Glosse dem Kommentar ähnlich ist und sich auf aktuelle Ereignisse bezieht, wird die Informationsfunktion realisiert. Der Autor hat die Absicht, die öffentliche Meinung zu beeinflussen, was mit der Appellfunktion gemeint ist.

\section{Zusammenfassung}

Zusammenfassend kann festgestellt werden, dass die Textsorte Glosse innerhalb der Jahre ihren Charakter völlig geändert hat. Zuerst wurde mit dem Begriff Glosse ein Teil der Zeitung oder Zeitschrift verstanden, der unter dem Strich platziert war. In diesem Ressort waren unterschiedliche Texte enthalten. Zuerst war es die Theaterrubrik, in der die Kritiken von Theaterstücken veröffentlicht wurden. 
Die Glosse diente vor allem der Kultur, deswegen wurden auch Buch-, Musikund später Filmkritiken verfasst. Um die Gewinne der Zeitung zu erhöhen, hat man im 18. Jahrhundert angefangen, in diesem Ressort Romane zu publizieren. Im 19. Jahrhundert hat die Glosse angefangen, eine völlig andere Rolle zu spielen. Sie diente der Kritik der Politik, des politischen Systems und der Regierenden. Das war für die Glossenautoren eine schwierige Zeit, weil sie oft in Konflikt mit dem Gesetz und den Regierenden geraten sind. Die Tatsache, dass Glossen den politischen Themen gewidmet sind, ist auch für die heutige Zeit kennzeichnend. Nur der Zweck hat sich etwas geändert. Die Glosse dient nämlich nicht nur der reinen Kritik, sondern auch der Unterhaltung. Der Themenbereich hat sich auch im Laufe der Zeit geändert. Jetzt werden die Texte nur selten der Kunst und Literatur gewidmet. Sie beziehen sich vor allem auf aktuelle Ereignisse, vor allem politischer, aber auch gesellschaftlicher und wirtschaftlicher Art.

\section{LITERATURVERZEICHNIS}

Frice H., Weimer K. (1997), Reallexion der deutschen Literaturwissenschaft, Walter de Gruyter, Berlin.

Henschied E. (1996), Tucholsky und die Spätfolgen. In: Literatur Konkret, Nr. 21, 220-234.

Jakoby R. (1998), Das Feuilleton des Journal des Débatas von 1814 bis 1830, Tübingen.

Linden P., Bleher C. (2002), Glosse und Kommentare in den Printmedien, Berlin.

Lüger H.-H. (1995), Pressesprache. 2. neu bearbeitete Auflage, Tübingen.

Nowag W., Schalkowski E. (1998), Kommentar und Glosse, Konstanz.

Oleze K. (1998), Das Feuilleton der Kölnischer Zeitung im Dritten Reich, Frankfurt/M.

Pietrzak M. (2013), Wyznaczniki gatunkowe felietonu drugiej połowy XIX wieku na przyktadzie tekstów Henryka Sienkiewicza, Bolesława Prusa i Aleksandra Świętochowskiego, Łódź.

Reus G. (1999), Ressort: Feuilleton, Konstanz.

Stöber R. (2000), Deutsche Pressegeschichte. Einführung, Systematik, Glossar, Konstanz.

Wildehann B. (2006), Feuilleton zwischen Kriegen. Die Formen der Kritik und ihre Theorie, Konstanz.

\section{Marta Wylot}

\section{THE TEXT TYPE ‘COLUMN’ IN THE DIACHRONICAL DEPICTION}

(Summary)

The aim of the paper is to describe the column as a type of text. This text type has its roots back in the $19^{\text {th }}$ century France. But at the beginning this text type had a totally different character, different form, aim and features. It was a part of newspaper, where novels, critiques and poems were posted. Nowadays there may occur a problem to define the term 'column'. Generally it is the text type with a polemical and derisive character, which serves as the entertainment, but sometimes the term 'column' still refers to a part of the newspaper. This article defines the term and how this text type has developed through the time.

Key words: the text type, the column, the text linguistic. 